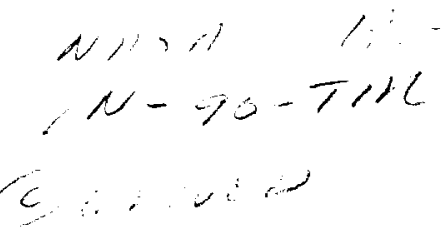

\title{
HIGH-DENSITY AMORPHOUS ICE, THE FROST ON INTERSTELLAR GRAINS
}

\author{
P. Jenniskens, D. F. Blake, M. A. Wilson, and A. Pohorille \\ NASA/Ames Research Center, Planetary Biology Branch, Space Science Division, Mail Stop 239-4, Moffett Field, CA 94035 \\ Received 1995 March 24 ; accepted 1995 June 15
}

\begin{abstract}
Most water ice in the universe is in a form which does not occur naturally on Earth and of which only minimal amounts have been made in the laboratory. We have encountered this "high-density amorphous ice" in electron diffraction experiments of low-temperature $(T<30 \mathrm{~K})$ vapor-deposited water and have subsequently modeled its structure using molecular dynamics simulations. The characteristic feature of high-density amorphous ice is the presence of "interstitial" oxygen pair distances between 3 and $4 \AA$. However, we find that the structure is best described as a collapsed lattice of the more familiar low-density amorphous form. These distortions are frozen in at temperatures below $38 \mathrm{~K}$ because, we propose, it requires the breaking of one hydrogen bond, on average, per molecule to relieve the strain and to restructure the lattice to that of low-density amorphous ice. Several features of astrophysical ice analogs studied in laboratory experiments are readily explained by the structural transition from high-density amorphous ice into low-density amorphous ice. Changes in the shape of the $3.07 \mu \mathrm{m}$ water band, trapping efficiency of $\mathrm{CO}, \mathrm{CO}$ loss, changes in the $\mathrm{CO}$ band structure, and the recombination of radicals induced by low-temperature UV photolysis all covary with structural changes that occur in the ice during this amorphous to amorphous transition. While the $3.07 \mu \mathrm{m}$ ice band in various astronomical environments can be modeled with spectra of simple mixtures of amorphous and crystalline forms, the contribution of the high-density amorphous form nearly always dominates.

Subject headings: dust, extinction — infrared: ISM : lines and bands - ISM : molecules - line: profiles methods: laboratory -- molecular data
\end{abstract}

\section{INTRODUCTION}

The frost on interstellar grains in dense molecular clouds is thought to consist mainly of an amorphous water ice with trapped impurities (e.g., Tielens \& Allamandola 1987). The observed $3.07 \mu \mathrm{m}$ absorption band is usually best compared to the $\mathrm{OH}$ stretch vibration band of impure water ice, vapordeposited onto inert surfaces at temperatures less than $30 \mathrm{~K}$ (Hagen, Tielens, \& Greenberg 1981; Schmitt et al. 1989). The same, or similar, ices are thought to constitute the bulk of comets (Mumma, Weissman, \& Stern 1993; Kouchi et al. 1994). The $3.07 \mu \mathrm{m}$ ice band has been studied in laboratory experiments of interstellar ice analogs as a function of temperature, deposition conditions, and impurity content (Hagen et al. 1981; Sceats \& Rice 1982. Hagen et al. 1983; Tielens, Hagen, \& Greenberg 1983; Schmitt, Ocampo, \& Klinger 1987). In addition, considerable effort has gone into measuring the changing shapes of infrared absorption bands of trapped impurities, the diffusion and recombination of radicals induced by UV photolysis, and the outgassing properties of ice mixtures (Bar-Nun, Herman, \& Laufer 1985; Bar-Nun et al. 1987; Grim \& Greenberg 1987; Laufer, Kochavi, \& Bar-Nun 1987; Sandford et al. 1988; Schutte 1988; Schutte, Allamandola, \& Sanford 1993).

The observed characteristics of vapor-deposited impure water ices are a function of the arrangement of the water molecules in the solid, which can be studied directly by electron or $\mathrm{X}$-ray diffraction. Fourier transformation of the observed diffraction patterns yields a statistical distribution of distances between the oxygen atoms in the water matrix (the oxygenoxygen radial distribution function), which uniquely characterizes its structure. By applying electron diffraction to dynamic systems, we find that vapor-deposited water ice exists in three amorphous forms, apart from two crystalline states (Jenniskens \& Blake 1994).
This paper characterizes the two low-temperature amorphous forms of vapor-deposited water ice, low-density amorphous ice $\left(I_{a} l\right)$ and high-density amorphous ice $\left(I_{a} h\right)$, and the behavior of these ices in the temperature range $15-120 \mathrm{~K}$. An amorphous form of water ice was first described early in this century. Diffraction studies were performed by Burton \& Oliver (1935). This familiar low-density form is made when water vapour is deposited on an inert surface at $77 \mathrm{~K}$. $\mathrm{I}_{\mathrm{a}} \mathrm{I}$ has a density of $0.94 \pm 0.03 \mathrm{~g} \mathrm{~cm}^{-3}$ (Narten, Venkatesh, \& Rice 1976; Mayer \& Pletzer 1986) and has been studied extensively because of its resemblance to liquid water (Olander \& Rice 1972; Sceats \& Rice 1982; Pohorille et al. 1987; Sciortino, Geiger, \& Stanley 1992), and because it can be used to replace liquid water as an embedding material for biological tissues in electron microscopy (Heide 1984; Heide \& Zeitler 1985).

High-density amorphous ice was first reported by Narten et al. (1976). X-ray diffraction patterns of water vapor deposited at $10 \mathrm{~K}$ on a single crystal copper substrate showed the presence of oxygen atoms at distances between those of the first and second nearest neighbor at the interstitial site of the $I_{a} l$ network, which results in a higher value for the calculated local density of oxygen atoms, i.e., $1.1 \pm 0.1$ instead of $0.94 \mathrm{~g} \mathrm{~cm}^{-3}$. For this reason, Narten et al. introduced the names of "high density" ( $I_{a} h-$ our notation) and "low density" $\left(I_{a} l\right)$ amorphous ice. Later experiments failed to reproduce the highdensity form on substrates other than single-crystal copper, and it was thought that the high-density amorphous form was an artifact of epitaxial growth on an oriented single crystal (Sivakumar, Rice, \& Sceats 1978; Sceats \& Rice 1982), depending, perhaps on a nucleation step (Sivakumar et al. 1977). Recently, it was shown that high-density ice also forms during vapor deposition on amorphous carbon films (Heide 1984; Jenniskens \& Blake 1994). However, it has not been shown that the resulting ice structure is the same as that of Narten 
et al. (1976). This high-density amorphous ice is potentially important in astrophysics, because infrared spectra of lowtemperature deposits provide the best fit to the $3.07 \mu \mathrm{m}$ ice band (Hagen et al. 1981). In the astrophysical literature, the high-density form has only briefly been referred to before by Hagen et al.

Another form of high-density amorphous ice can be made by pressure melting, and is similarly referred to as high-density amorphous ice (hda or HDA). When cooled to $77 \mathrm{~K}$, crystalline hexagonal ice, $I_{h}$, undergoes a transition into hda at 1-2 GPa pressure (Mishima, Calvert, \& Whalley 1984, 1985). The resulting ice, when recovered at $1 \mathrm{~atm}$, has a density of $1.17 \pm 0.03 \mathrm{~g} \mathrm{~cm}^{-3}$ (determined by weighing in liquid nitrogen). Theoretical estimates of the transition pressure as a function of temperature are given in Tse \& Klein (1990) and Poole et al. (1992). HDA can exist metastably for some time at low pressure and $77 \mathrm{~K}$. When this pressure-induced, highdensity amorphous ice is warmed at a rate of $0.2-3 \mathrm{~K}$ minutes $^{-1}$, the ice transforms irreversibly into a low-density $0.94 \pm 0.02 \mathrm{~g} \mathrm{~cm}^{-3}$ amorphous form (lda or LDA) in the range 105-123 K, releasing energy in the process (Mishima et al. 1984, 1985; Handa, Mishima, \& Whalley 1986; Bizid et al. 1987; Tse \& Klein 1987; Hemley, Chen, \& Mao 1989). The radial distribution function of hda (Bosio, Johari, \& Teixera 1986; Bizid et al. 1987) differs significantly from that of the $I_{a} h$ of Narten et al. (1976). It has been argued that pressureinduced hda has more hexagonal order than vapor-deposited I $h$ (Tse 1992).

In this paper, we confirm the existence of the vapordeposited, high-density amorphous form $I_{a} h$ from electron microscopy studies $(\$ 2)$. We measured the radial distribution function (rdf) of $\mathrm{I}_{\mathrm{a}} \mathrm{h}$ and the gradual change of the diffraction pattern during the $I_{a} h \rightarrow I_{a} l$ transition $(\S 3, \S 4)$. The rdf is compared to that of pressure-induced hda and similar data by Narten et al. (1976). The distinct physical properties of $I_{a} h(\$ 5)$ and the conditions necessary for formation during vapor deposition $(\S 6)$ are determined by reinterpreting experimental data reported in the literature. In $\S 7$, new molecular dynamics calculations are presented that attempt to model the ultrastructure of high-density amorphous ice. The theoretical and experimental results are discussed in $\$ 8$. In $\$ 9$, it is argued that high-density ice is ubiquitous in space, to an extent that this form is probably the most abundant form of ice in the universe.

\section{CHARACTERIZING THE STRUCTURE OF AMORPHOUS ICES}

The electron diffraction studies employ a Hitachi $\mathrm{H}-500 \mathrm{H}$ transmission electron microscope (TEM), which was adapted for vapor-depositing water molecules in situ onto a thin amorphous carbon film subtrate held at a temperature of $14 \mathrm{~K}$ (Blake \& Palmer 1991). The structure of the ice film is characterized by selected area electron diffraction (SAED). Morphological features of the ice can be viewed in either bright-field mode (strongly diffracting regions of the film appear dark) or dark-field mode (diffracting regions appear bright).

Degassed distilled water vapor is deposited from a reservoir at 14 torr and room temperature into the microscope at base pressure of $\sim 3 \times 10^{-7}$ torr, until the partial $\mathrm{H}_{2} \mathrm{O}$ pressure increases to $\sim 4 \times 10^{-6}$ torr. A film of ice is grown by opening a shutter that covers the sample for 5-20 s (rate of growth 6-36 $\mu \mathrm{m} \mathrm{hr}^{-1}$ ), while monitoring the diffraction pattern. The resulting film is about $0.05 \mu \mathrm{m}$ thick, smooth, and homogeneous. Bright-field imaging reveals no sign of the crystallinity reported by Olander \& Rice (1972), even at high magnification. There is also no indication of needle- or stalactite-like surfaces as reported by Laufer et al. (1987).

Diffraction patterns from the ice film are obtained from volumes of material of about $0.7 \mu \mathrm{m}^{3}$. A parallel eletron beam of $3 \mu \mathrm{m}$ in diameter is directed through the sample to form the diffraction pattern, which is focused onto an imaging device at the back focal plane of the microscope. The resulting diffraction pattern is a Fourier transform of the statistical distribution of distances between atoms in the ice. Oxygen atoms are the principal electron scatterers (scattering by hydrogen atoms is negligible). The diffraction pattern is electronically recorded with an instrumental resolution of $\Delta k=0.08 \AA^{-1}$, sampled at $0.03 \AA^{-1}$ pixel $^{-1}$, and the patterns are stored at a rate of $1-3$ per minute during a typical experiment. Temperatures can be recorded with an absolute accuracy of $\pm 2 \mathrm{~K}$ and relative accuracy of about $0.2 \mathrm{~K}$. The warming rate in these experiments varied from $2.3-5.2 \mathrm{~K}$ minutes $^{-1}$ and was typically $2.7 \mathrm{~K}$ minutes $^{-1}$.

The two-dimensional circular diffraction patterns are reduc ed by folding to a one-dimensional radial intensity distribution (Fig. 1). The $x$-axis, the momentum transfer coordinate $k=(4 \pi / \lambda) \sin \vartheta$, is calibrated using crystalline gold $\left(a_{0}=\right.$ $4.0786 \AA$ ) deposited on a small part of the substrate as an internal standard, which was measured at the beginning and end of each experiment. The relatively small intensity contributed by the amorphous carbon substrate is removed by a background subtraction algorithm.

Low electron dose imaging techniques are employed to minimize sample degradation during viewing. Electron beam damage does not change the diffraction patterns on the timescale of each measurement, and different parts of the ice layer are exposed during each subsequent measurement. The technique offers a powerful means of measuring the structural properties of water ice films in situ in vacuum.

Fourier transformation of the diffraction patterns yields the rdf of distances between oxygen atom pairs (Fujime 1966;

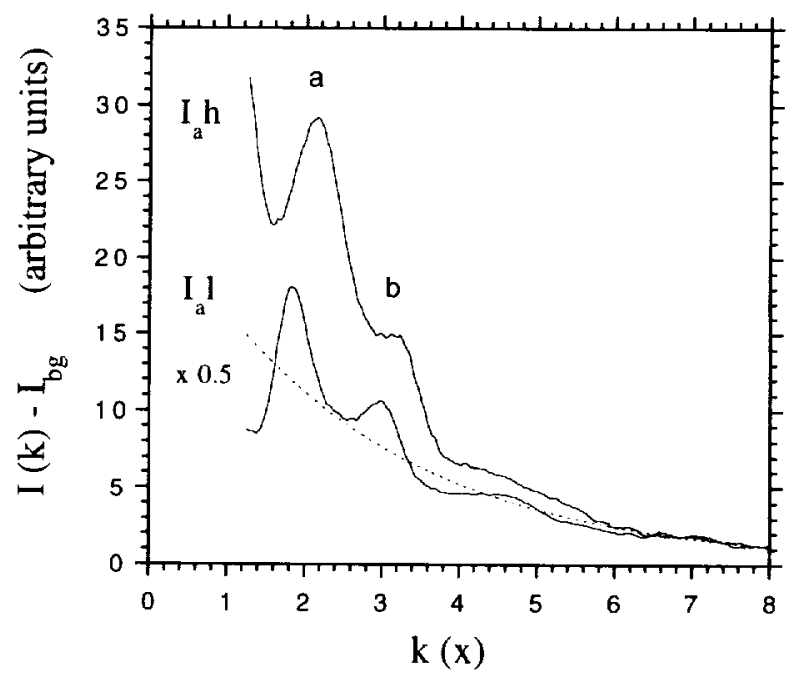

FIG. 1.-Representative electron diffraction patterns of high-density $(I, h)$ and low-density $\left(I_{\mathrm{a}} \mathrm{l}\right)$ amorphous ice, at 14 and $77 \mathrm{~K}$, respectively, after subtraction of a contribution by the amorphous carbon substrate. The dashed line is an example of the chosen background function in the derivation of the radial distribution function ( $I_{\mathrm{bg}}$ in eq. 1). The first and second diffraction maxima are labeled " $a$ " and " $b$ " and are referred to in Fig. $3 a-3 c$ 
Moine, Pelton, \& Sinclair 1988)

$$
\begin{aligned}
k & =(4 \pi \sin \vartheta) / \lambda, \\
I(k) & =\left[I_{\mathrm{obs}}(k)-I_{\mathrm{bg}}(k)\right] / \beta f_{\mathrm{O}}^{2}, \\
\mathrm{rdf} & =(2 / \pi) \int_{0}^{k_{\max }} I(k) \exp \left(-\alpha k^{2}\right) \sin (k r)(k / r) d k,
\end{aligned}
$$

where $I_{\text {obs }}$ is the observed diffraction pattern after subtraction of a substrate contribution, $I_{\mathrm{bg}}$ is a smooth and continuous function through the inversion points of the pattern (a sum of three power laws; Fig. 1), $\beta$ is a scaling factor taken such that the intensity is -1 for small $k$ after subtraction of the background (at onset of first diffraction peak), and $f_{0}$ is the electron scattering function of oxygen. The parameter $\alpha$, in the damping factor in the Fourier transform, is taken such that exp $\left(-\alpha k_{\max }^{2}\right)=0.1$. The latter reflects the rapid decrease of the electron beam intensity away from the center of the beam and the limited range of $k$ that is measured.

Due to limitations in the dynamic range of our electronic imaging system, we cannot derive an rdf for every recorded pattern. In order to follow the changes in the structural state, we measure the position, height, and width of each peak in the diffraction pattern by fitting Gaussian profiles to individual maxima. The Gaussian profiles usually produce a good fit to the peaks, which is the only and sufficient reason for choosing this particular functional form.

\section{THE RADIAL DISTRIBUTION FUNCTION OF $I_{a} h$ AND $I_{a} l$}

When water ice is formed by yapor deposition at $14 \mathrm{~K}$, it yields a distinct diffraction pattern when compared to that of the more familiar low-density amorphous ice at $77 \mathrm{~K}$ (Fig. 1). Most notably, the first diffraction peak is shifted to larger values of $k$.

The resulting radial distribution function of the lowtemperature deposit (Fig. 2), which Narten et al. (1976) calls "high-density amorphous ice" $\left(\mathrm{I}_{\mathrm{a}} \mathrm{h}-\right.$ our notation), differs principally from the higher temperature deposit, $I_{\mathrm{a}} \mathrm{l}$, in having an additional maximum between 3 and $4 \AA$.

It is illustrative to compare the radial distribution functions of $I_{a} h$ and $I_{a} l$ to those of vapor-deposited amorphous ice at 10 $\mathrm{K}$ (Narten et al. 1976), and those of pressure amorphosed hexagonal ice (Bizid et al. 1987). Figure 2 shows that there is a striking similarity between our $\mathrm{rdf}$ of vapor-deposited $\mathrm{I}_{\mathrm{a}} \mathrm{h}$ and that of the pressure-induced $\mathrm{D}_{2} \mathrm{O}$ ice by Bizid et al. (1987). The high-density amorphous form is characterized, in both cases, by a splitting of the second nearest neighbor peak, such that one component lies between the first and second nearest neighbor peaks of the low-density amorphous form. The diffraction maxima in $I_{a} h$ are less pronounced, suggesting that $I_{a} h$ is less well ordered than Bizid's hda. This apparent reduction in ordering may be due to the thinness of the vapor-deposited ice, differences in annealing times between the two ices, exposure to high fluxes of X-rays, or more microporosity in the vapordeposited ice (that is, defects in the random hydrogen-bonded network with typical cavity sizes of radius $1 \AA<r<10 \AA$ ).

The agreement with the pressure-induced hda is better than with the vapor deposit of Narten et al. (1976). The first nearest neighbor distance of both $I_{a} h$ and $I_{a} l$ is $2.81(+0.05 /-0.01) \AA$, in good agreement with the hda of Bizid et al. (1987) $(2.80 \AA)$ and Bosio et al. (1986) $(\sim 2.82 \AA)$, while Narten et al. (1976) report a slightly smaller value at $2.76 \AA$. The second nearest neighbor in our $I_{a} h$ is broad and is split into two peaks at
$3.70 \AA$ and $4.70 \AA$, similar to the hda of Bizid et al., 3.70 and $4.64 \AA$; and that of Bosio et al., 3.65 and $4.45 \AA$, respectively. However, neither our, nor Bizid's, radial distribution functions show a sharp spike at the "interstitial" position as in the Narten et al. (1976) data.

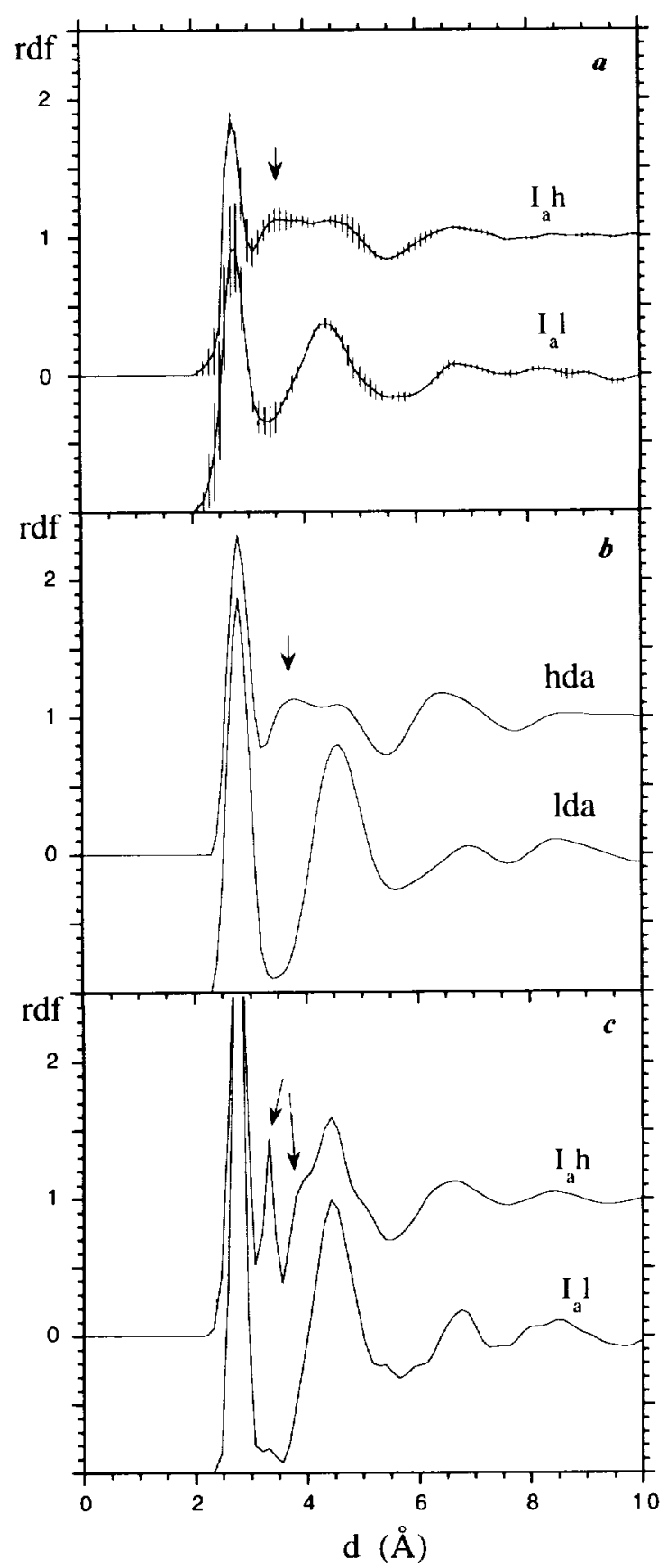

FIG. 2,-Fourier transform of the diffraction pattern. These oxygen-oxygen distance radial distribution functions ( $r$ df's) depict the probability of finding another oxygen atom at given radial distance. The rdf of low-density ice is displaced by -1.0 for clarity. From top to bottom: (a) rdf derived from our electron diffraction data of vapor-deposited ice at $15 \mathrm{~K}$ (top) and after warm-up to $77 \mathrm{~K}$ (bottom); (b) X-ray diffraction data of the pressure-induced high- and low-density ice at $77 \mathrm{~K}$ by Bizid et al. (1987) directly after pressure relief and after a brief warming to $140 \mathrm{~K}$ and subsequent cooling, respectively; and $(c)$ as derived by Narten et al. (1976) from X-ray data of vapor-deposited water at $10 \mathrm{~K}\left(\mathrm{I}_{\mathrm{a}} \mathrm{h}\right)$ and of a vapor deposit at $77 \mathrm{~K}\left(\mathrm{I}_{\mathbf{a}} \mathrm{l}\right)$. 
also be identified indirectly. We have reinterpreted experimental data reported in the literature, in light of the previous results, and suggest that the high-density amorphous form can be identified by the shape of the $3.07 \mu \mathrm{m}$ band (Hagen et al. 1981; Mayer \& Pletzer 1985), the shape of the dangling $\mathrm{OH}$ bond absorption bands (Venkatesh, Rice, \& Bates 1975; Sivakumar et al. 1978), the presence of CO impurities (Sandford et al. 1988; Schmitt, Greenberg, \& Grim 1989), outgassing behavior (Laufer et al. 1987; Kouchi 1990), and the diffusion of radicals (Schutte 1988).

Figure 4 summarizes the deposition conditions for the experiments cited above. It is concluded that $I_{a} h$ forms readily when water is condensed slowly $\left(<100 \mu \mathrm{m} \mathrm{hr}{ }^{-1}\right)$ at low enough temperature $(T<40 \mathrm{~K})$ on any surface with good thermal conductivity (single-crystal copper, amorphouscarbon film, aluminum, gold-coated copper, CsI window). Vapor deposits at 40-70 K form mostly $I_{a} I$ (see, e.g., Sandford et al. 1988).

There is a maximum and a minimum condensation rate at which $I_{a} h$ can form. Deposition rates close to the maximum of $100 \mu \mathrm{m} \mathrm{hr}^{-1}$ do result in a structure with more $I_{\mathrm{a}} l$ character. For example, Palumbo \& Strazzulla (1993) measured a noticeable change in the position of the $\mathrm{C}-\mathrm{O}$ stretch in an impure water ice for deposition rates above $1 \mu \mathrm{m} \mathrm{hr}^{-1}$. For a given temperature, there is, potentially, a minimum deposition rate, below which the water molecules have time to reorient themselves before being incorporated in the $I_{a} h$ lattice by subsequent condensing molecules. Kouchi et al. (1994) measured this effect for the formation of the cubic crystal form in the low-density amorphous domain at very low deposition rates, and found a temperature dependence, which, to us, suggests a characteristic $\Delta H=44 \pm 7 \mathrm{~kJ} \mathrm{~mol}^{-1}$ activation enthalpy. This value is the same as that for the crystallization of $I_{a} l$ during warm-up (Jenniskens \& Blake 1994). An extrapolation of the measured critical flux at 96-110 K (Kouchi et al. 1994) down to

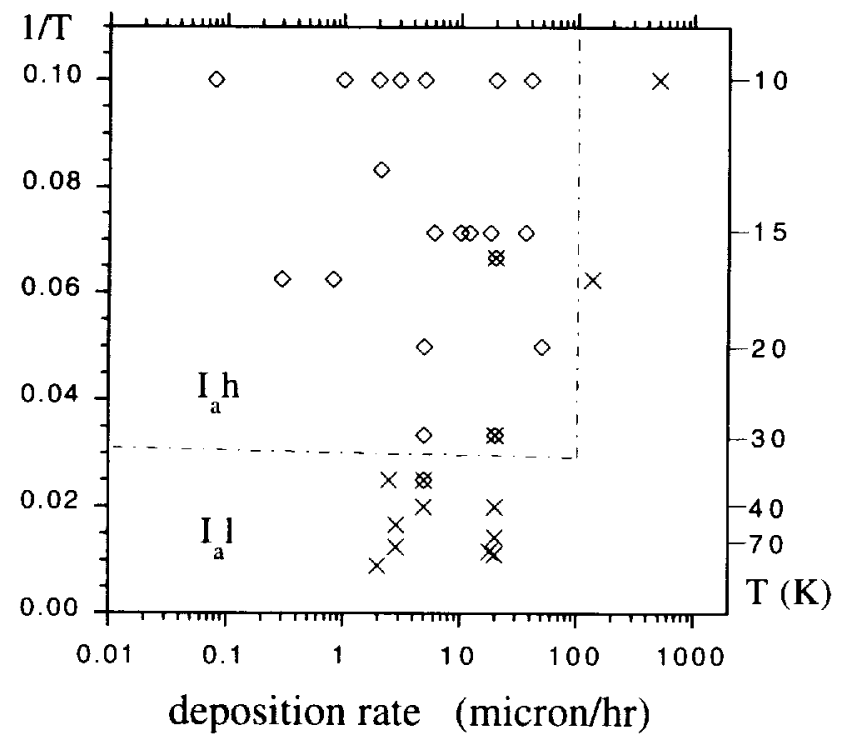

Fıg. 4.-Deposition conditions that are favorable for the formation of $1, h$ (diamond) and $I_{2} l$ (cross), as inferred from five deposition experiments at $14 \mathrm{~K}$ and from direct and indirect indicators in 23 literature studies. A combination of diamond and cross indicates a weak high-density amorphous ice signature only. The one at $T=15 \mathrm{~K}$ is a thick $1 \mathrm{~mm}$ ice layer by Venkatesh, Rice, \& Bates (1975). The dashed lines are approximate boundaries discussed in the main text. lower temperature suggests that, at realistically low deposition rates, no cubic crystalline ice will be formed in the high-density regime below $40 \mathrm{~K}$. However, a lower activation energy may apply for the reorientation of the water into an open $\mathrm{I}_{\mathrm{a}} \mathrm{I}$ structure. The horizontal line in Figure 4 shows this deposition rate dependence, if the characteristic activation enthalpy is $12 \mathrm{~kJ}$ $\mathrm{mol}^{-1}$ (see $\S 8.2$ ). The result suggests that this effect plays no significant role in the laboratory experiments, but may be relevant in case of extremely low deposition rates in some astrophysical environments.

The film thickness has an effect as well. Most experiments concern layers of $0.01-10 \mu \mathrm{m}$ in thickness. Low-temperature vapor deposits as thick as $1000 \mu \mathrm{m}$ sometimes do (Narten et al. 1976), and sometimes do not, show $I_{a} h$ signature (Venkatesh et al. 1975). Perhaps this is because in thicker films the heat of fusion from impinging molecules is not conducted fast enough to prevent local restructuring. There is also some indication that for very thin layers $(<0.002 \mu \mathrm{m})$, substrate surface structure can affect the structural growth during deposition (Moore et al. 1994) and, therefore, the presence of a surface film on the substrate, and the existence of single-crystal orientations of the substrate, may affect the structure of thin deposits through epitaxy or nucleation (Sceats \& Rice 1982).

Finally, we note that the $I_{a} h \rightarrow I_{a} l$ transition does not always unfold the same way in vapor-deposited layers. For example, Hagen et al. (1981) studied a $0.5 \mu \mathrm{m}$ thick water ice layer, vapor-deposited at $10 \mathrm{~K}$. We assume that the observed shift of the peak frequency and width of the infrared $\mathrm{O}-\mathrm{H}$ stretching band is due to the $I_{a} h \rightarrow I_{a} I$ transition, and find that the transition had an onset shortly after the beginning of the warm-up at about $20 \mathrm{~K}$, which is at lower temperature than in our data. The transition was complete at about $70 \mathrm{~K}$, as in our data. On the other hand, the $1000 \mu \mathrm{m}$ thick ice layer of Narten et al. (1976) still had a recognizable "interstitial" peak in the rdf after warming to $77 \mathrm{~K}$.

\section{MODEL CALCULATIONS}

Molecular dynamics (MD) calculations of water structure, that is, solutions of the equations of motion for an ensemble of point masses with the intermolecular potentials of water, go back two decades (for reviews see Angell 1983 and Dore \& Teixeira 1991). The resulting structures are best described as an imperfect random network of hydrogen bonds. The models successfully describe many features of the measured structure and physical properties of liquid water and low-density ice $I_{a} l$.

\subsection{Vapor Deposition}

MD simulations of water vapor deposition at $10 \mathrm{~K}$ on small water clusters (Buch 1992) or on films (Wilson et al. 1995) do not yield $\mathrm{I}_{\mathrm{a}} \mathrm{h}$. Instead, the resulting network is found to contain a substantial fraction of molecules with hydrogen bond coordination different from $4(1,2,3$ and 5) and an abundance of dangling $\mathrm{OH}$ bonds. This results in a porous structure with many microvoids and a very large effective surface area. Buch (1992) ascribed this behavior to the preferred attachment of new molecules to dangling atoms on the surface (unbonded $\mathrm{H}$ atoms) and to $\mathrm{O}$ atoms with only one hydrogen bond, thus propagating the formation of surface protrusions. MD calculations are limited by the amount of relaxation that can be allowed after a molecule has collided with the surface. The ensemble can usually only be followed for tens of picoseconds before allowing the next molecule to hit the surface. In effect, this corresponds to deposition rates of $10^{8}-10^{9} \mu \mathrm{m} \mathrm{hr}^{-1}$. The 


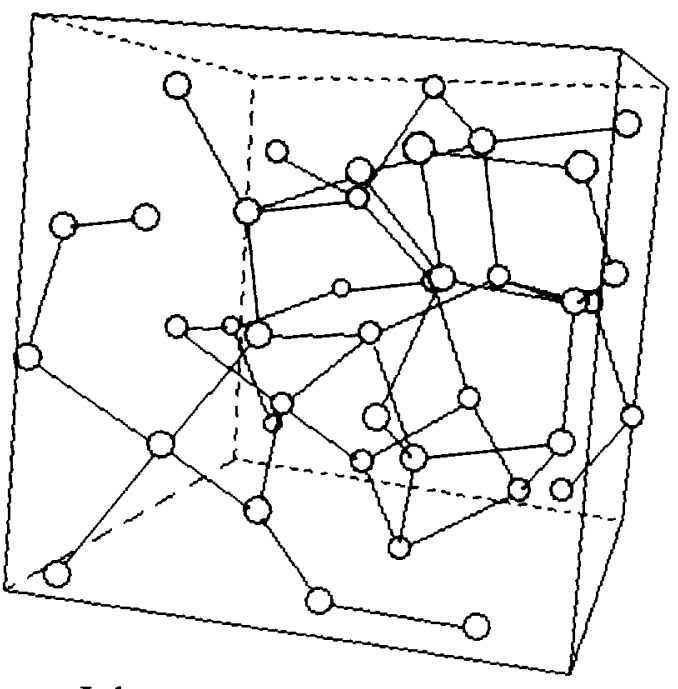

Iah

Fig. $5 a$

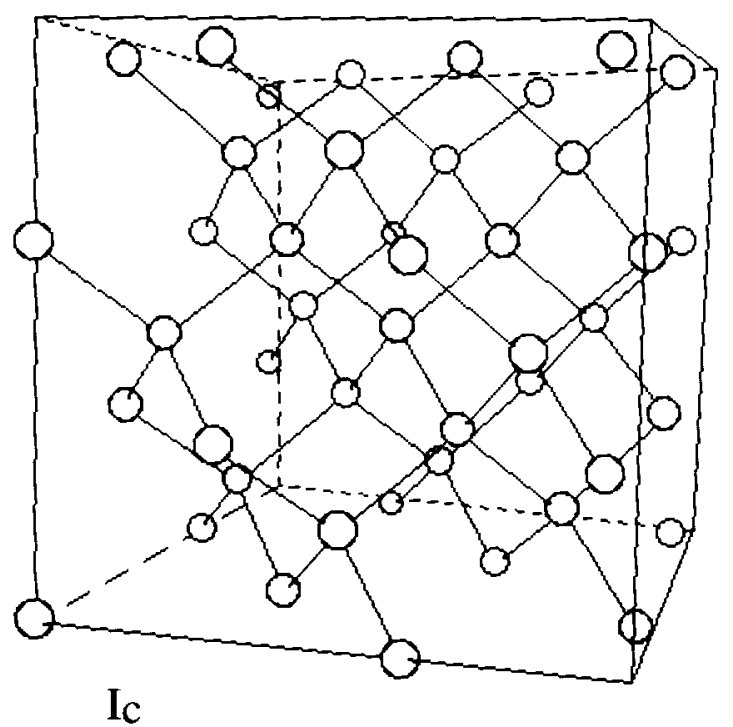

Fig. $5 c$

Fig. 5.-Model of water ice. A $10 \times 10 \times 10 \AA^{3}$ sample from a MD simulation of (a) high-density amorphous ice $(b)$ low density amorphous ice, and $(c)$ cubic crystalline ice. These stick diagrams are randomly chosen from one final configuration of a 340 molecule model and show the position of the oxygen atoms only. The van der Waals radius of the water molecules is larger than the size of the symbols. Solid lines connect the oxygen atoms that are less than 3.0 $\AA$ apart. For comparison purposes, the cubic ice is shown at an unfavorable angle with respect to the crystal lattice planes and axis of symmetry.

molecular velocities within the cluster are rescaled, after each sticking trajectory, by a constant factor to represent cooling back to the initial temperature of $10 \mathrm{~K}$. However, this quenching may not result in the same final state as a fully relaxed network. In addition, the water molecules are considered rigid and certain energy transfer pathways are not considered in the calculations, including quantum mechanical tunneling and energy transfer to intramolecular $\mathrm{O}-\mathrm{H}$ stretch vibrations. Note that the energy of binding is of similar magnitude as

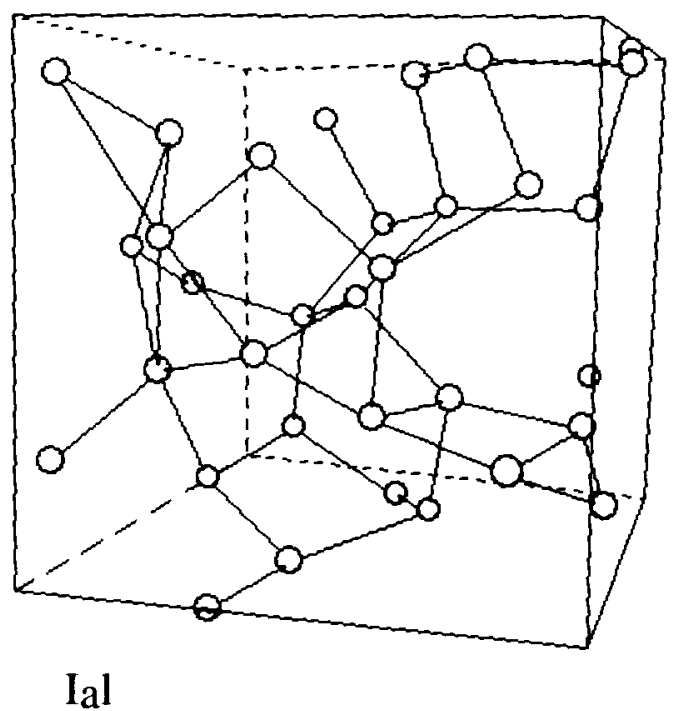

FIG. $5 b$

the energy of an intramolecular $\mathrm{O}-\mathrm{H}$ stretch vibration or bending mode.

\subsection{Pressure Melting}

MD simulations have modeled the transition of hexagonal crystalline ice $\left(\mathrm{I}_{h}\right)$ into high-density amorphous ice by pressure (Tse \& Klein 1987; Tse 1992). The process was found to be driven by a collapse of the open hexagonal ice $I_{h}$ structure. In the resulting oxygen-oxygen rdf, the second nearest neighbor peak, at $4.5 \AA$, broadens significantly, and a new band, at $3.2 \AA$, starts to emerge, reflecting a migration of water molecules into the interstitial sites. A number of hydrogen bonds are broken, creating 3-coordinated and 5-coordinated molecules. Some memory of $I_{h}$ remains: the network appears to keep a recognizable layering (stacking order) and 6-fold symmetry when viewed along the corresponding crystal axis (Tse 1992).

\subsection{Quenching Configurations of Liquid Water}

We have attempted to model high-density amorphous ice by quenching configurations, drawn from MD simulations of liquid water in a confined space at $300 \mathrm{~K}$, instead of a crystalline $I_{h}$ configuration, therefore starting from an amorphous configuration. The system consisted of 343 water molecules placed in a cubic simulation box, assuming periodic boundary conditions and imposing a density of $1.17 \mathrm{~g} \mathrm{~cm}^{-3}$. Instead of a steepest descent minimization of the high-density water configurations, as in our previous model (Wilson et al. 1995), a more gentle quenching was attempted. Each stage of quenching consisted of a 20 ps trajectory during which the temperature was reduced by $50 \mathrm{~K}$, followed by 20 ps of equilibration. Most of the network changes occurred in the higher temperature regime above $150 \mathrm{~K}$. Subsequently, at $77 \mathrm{~K}$, the simulation box was expanded in one dimension, thereby creating a slab of ice with two free surfaces. The structure did not change on a tens of picoseconds timescale when allowed to expand in two dimensions.

To illustrate the disordered nature of these amorphous ices, Figures $5 a-5 c$ show a small $10 \times 10 \times 10 \AA$ cube of the highdensity amorphous, low-density amorphous, and crystalline $I_{c}$ configurations. The planes of molecules, and the cubic and 
hexagonal symmetries that are typical of the crystalline polymorphs, cannot be recognized in the amorphous form when viewed from different angles. There are many 4-, 5-, and 7ringed structures which create a wide range of cavities or "interstitial" sites.

The calculated oxygen-oxygen rdf of the high-density amorphous ice, at $77 \mathrm{~K}$, shows a broad second nearest neigh-

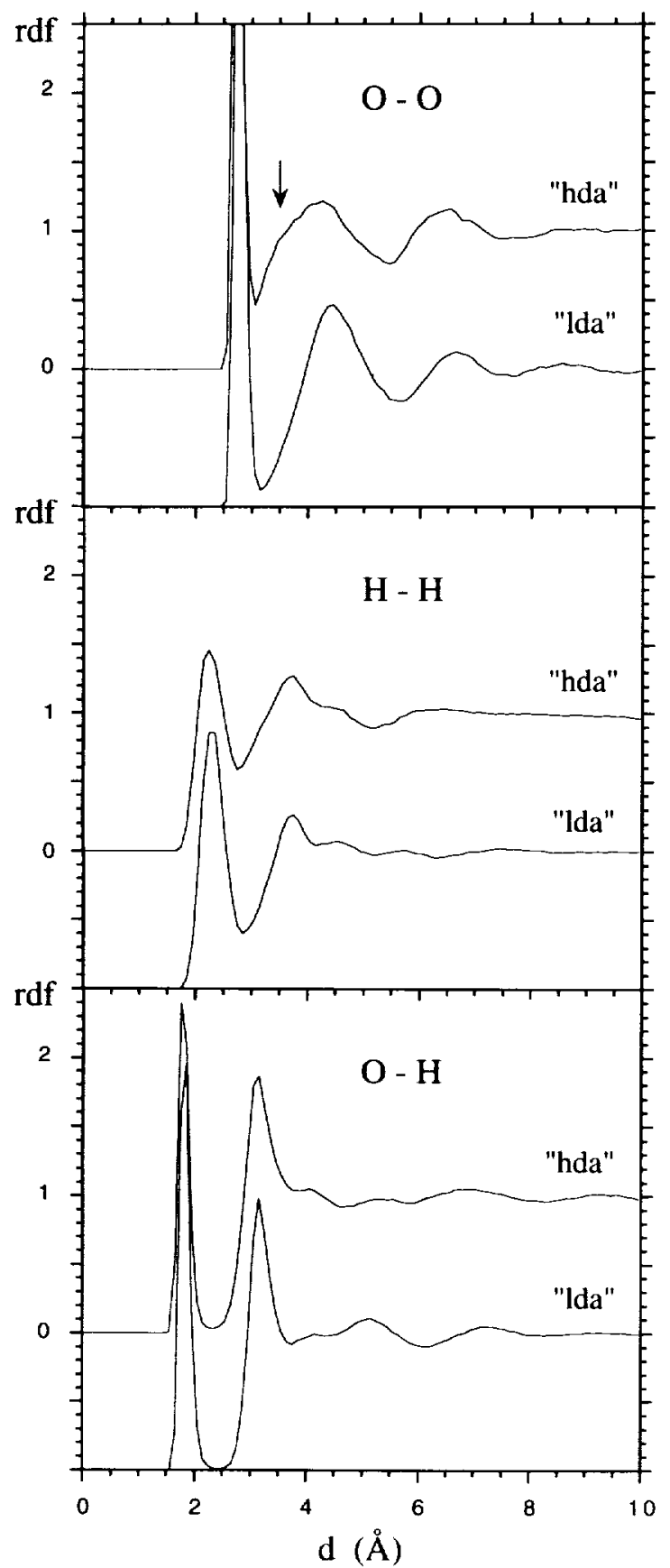

Fig. 6.-Radial distribution functions, averaged from 50 such configurations of high- and low-density ice as shown in Fig. 5. The oxygen-oxygen rdf can be compared to those in Fig. $2: I_{\mathrm{a}} \mathrm{h}$ has a broad second nearest neighbor peak, with a shoulder at $3.7 \mathrm{~A}$ that is not found in $\mathrm{I}_{\mathrm{a}} \mathrm{l}$. The hydrogen-hydrogen rdf (only intermolecular distances) shows the same pattern of bond distances. There is no clear difference between high- and low-density ice in the oxygenhydrogen rdf (again only intermolecular distances). bor oxygen peak, with some indication of splitting into two components (Fig. 6). The peak is not as pronounced as in the collapsed $I_{h}$ model of Tse \& Klein (1987), but is clearly different from the rdf of the low-density ice model. Also, this peak spans the region between the "interstitial" and second nearest neighbor peak in the observed rdf of $I_{a} h$ (Fig. 2).

These models of $I_{a} h$ and $I_{a} l$ were investigated further by calculating the distribution of coordination numbers and the distribution of cavity sites in the ice, as in Pratt \& Pohorille (1992). The coordination number (Table 2) is defined in two ways: either by the number of oxygen atoms within a sphere of $3.2 \AA$, or by the presence of a hydrogen bond stronger than -8 $\mathrm{kJ} \mathrm{mol}^{-1}$. The number distribution of hydrogen bonds shows that (1) there are no interstitial water molecules with zero hydrogen bonds; (2) the most preferred coordination is 4 ; (3) the distribution of low coordination numbers less than 4 does not differ between the high and low-density amorphous ice models; (4) in high-density amorphous ice, there is a large percentage of molecules with five, six, and even seven oxygen atoms located within $3.2 \AA$, that are not found in low-density ice; (5) the increased percentage of short oxygen-oxygen distances does not correspond to a large number of 5-coordinated hydrogen bonds, although the percentage of 5-coordinated water is somewhat higher in high-density ice.

Figure 7 shows the probability of placing a rigid sphere of given radius at a random location in the ice structure (allowing for the Van der Waals radius of the water molecules). Large open spaces are conspicuously absent in high-density amorphous ice, relative to low-density amorphous ice. For radii larger than $0.3 \AA$, there is a fast decline of the relative probability of placing a cavity of given radius at given position. In the model of high-density amorphous ice, cavities larger or equal to the largest cavity in the cubic crystalline lattice are highly improbable.

\section{DISCUSSION}

\subsection{A Model of High-Density Ice}

Two models have been proposed for vapor-deposited highdensity ice: (1) unbonded water molecules are trapped in interstitial sites in slightly distorted ice I cages, in which $45 \%$ of the cavities are filled with interstitial water, or $19 \%$ of all water molecules are in cavity positions (Narten et al. 1976); and (2) high-density amorphous ice is a randomized version of ice II or III, high-pressure crystalline polymorphs of water ice which

TABLE 2

Percentile Distribution of Oxygen Coordination Numbers"

\begin{tabular}{|c|c|c|c|c|c|c|}
\hline \multirow{2}{*}{$\begin{array}{l}\text { COORDINATtON } \\
\text { NumBER }\end{array}$} & \multicolumn{3}{|c|}{$0-0<3.2 \AA$} & \multicolumn{3}{|c|}{$\mathrm{H}$ Bond $<-8 \mathbf{k J ~ \mathrm { mol } ^ { - 1 }}$} \\
\hline & hda & Ida & $I_{c}$ & hda & Ida & $I_{c}$ \\
\hline 0. & 0.00 & 0.00 & 0.00 & 0.26 & 0.18 & 0.00 \\
\hline & 0.00 & 0.00 & 0.00 & 4.9 & 4.4 & 0.00 \\
\hline …… & 0.02 & 0.13 & 0.00 & 17.1 & 16.3 & 0.00 \\
\hline 3.. & 2.5 & 6.9 & 0.00 & 28.3 & 28.4 & 0.00 \\
\hline 4. & 58.8 & 86.4 & 100.00 & 43.9 & 48.7 & 100.00 \\
\hline$\ldots \ldots \ldots \ldots$ & 30.3 & 6.4 & 0.00 & 5.4 & 2.00 & 0.00 \\
\hline $6 \ldots \ldots \ldots \ldots \ldots$ & 7.3 & 0.15 & 0.00 & 0.08 & 0.00 & 0.00 \\
\hline $7 \ldots \ldots \ldots \ldots$ & 0.06 & 0.003 & 0.00 & 0.00 & 0.00 & 0.00 \\
\hline
\end{tabular}

- Based on the number of oxygen atoms within a radius of $3.2 \AA$ and the number of those oxygen atoms that have a hydrogen bond strength larger than $-8 \mathrm{~kJ} \mathrm{~mol}^{-1}$ (statistical error is \pm 2 in the least significant digit). 


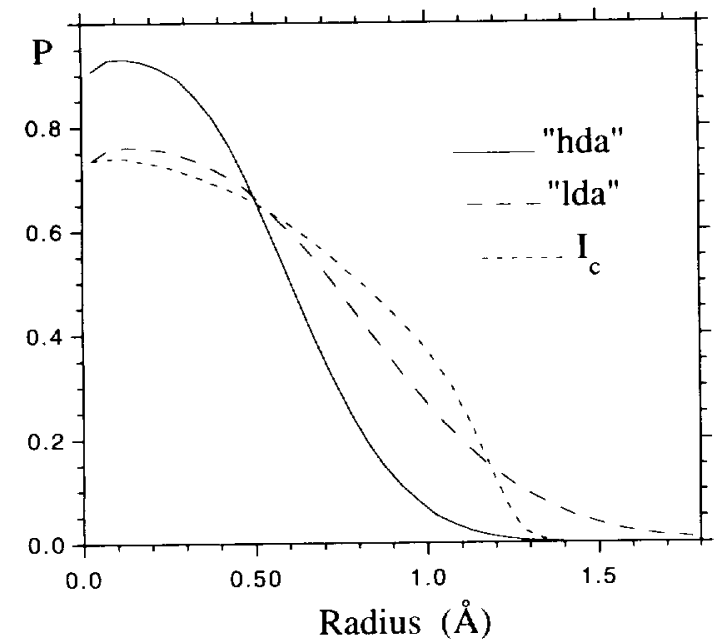

FIG. 7.- The probability of placing a rigid sphere of given radius at a random location in the free space of the water ice model, allowing for the van der Waals radius of the water molecules. The three curves are for the models of the two low-temperature amorphous forms and the crystalline cubic lattice. The high-density amorphous form has a significant lack of the large cavities found in the low-density amorphous form.

have a similar mean density (Narten et al. 1976; Sceats \& Rice 1982).

These models are highly unlikely in a distorted network. Some bonding of the interstitial water to other water molecules is expected. The presence of a distorted form of one of the high-pressure crystalline polymorphs of ice is also, in our opinion, not reasonable, because either these polymorphs need a form of proton ordering (preferred orientation of the water molecules), or they are outside their stability regime.

Following Tse \& Klein's interpretation of the difference between hda and lda (Tse \& Klein 1987; Tse 1992), we propose an alternative model: the high-density amorphous form is a collapsed low-density lattice, characterized by the presence of oxygen-oxygen distances between 3 and $4 \AA$. Arguments for using the term "collapsed" include that the number density of the large cavity sites is significantly reduced in high-density amorphous ice compared to the low-density ice. Also, there are more cases of 5 oxygen atoms within $3.2 \AA$ of another oxygen atom, without a similar increase in the number of 5coordinated hydrogen bonds.

This raises the question of how a collapsed lattice is formed during vapor deposition. Indeed, MD model calculations typically result in the formation of an open $I_{a} l$ lattice. There is a possibility that ice is deposited in an $\mathrm{I}_{\mathrm{a}} \mathrm{h}$ form because nature offers a faster redistribution of the energy of binding (some 30 $\mathrm{kJ} \mathrm{mol}{ }^{-1}$ on average) than the molecular dynamics calculations allow for (e.g., because in the MD model the water molecules are rigid). Alternatively, the ice may deposit itself in an open $I_{a} l$ form, but a collapse to $I_{a} h$ occurs below the binding site upon release of binding energy, analogous to the injection of energy in the ice network, as in charged particle bombardment and photolysis $(\$ 4)$, while the MD calculations, again, do not allow a fast enough dissipation of energy for the distortion to be frozen in.

There is no single structure which characterizes $I_{a} h$. Instead, a wide range of configurations is possible, depending on the number of collapsed cages and the amount of relaxation toward an $I_{a} l$ structure. Such relaxation apparently causes the less distorted cages to be restructured more easily than the more heavily distorted structures, with water molecules at an "interstitial" position in an ice $I_{a} l$ lattice. The difference between hda and $I_{a} h$, if any, is that hda may have a more recognizable hexagonal stacking order than $I_{a} h$.

\section{2. $I_{a} h \rightarrow I_{a} l$ and $I_{a} l \rightarrow I_{a} h$}

The presence of a well-defined transition between one amorphous form and the other suggests that there is an activation energy barrier that prevents the transition at temperatures below $38 \mathrm{~K}$ in a vapor deposit, and significantly delays the transition below $70-80 \mathrm{~K}$ in electron-irradiated $\mathrm{I}_{\mathrm{a}} 1$ or $\mathrm{I}_{\mathrm{c}}$. The nature of this barrier determines the physical difference between the two amorphous forms.

We speculate that the $I_{a} h \rightarrow I_{a} l$ transformation requires the breaking of one hydrogen bond, on average, per molecule in the $I_{a} h$ structure. An argument for this is that the temperature range of $70-90 \mathrm{~K}$ that discriminates the two regimes is at half the temperature needed to transform amorphous ice into cubic ice. The latter transition occurs at 143-155 $\mathrm{K}$ and has a characteristic activation enthalpy of $\Delta H_{a}=44 \pm 2 \mathrm{~kJ} \mathrm{~mol}^{-1}$ (Jenniskens \& Blake 1994), which is equivalent to breaking 2 hydrogen bonds on average per molecule. The onset of the transition will scale with the activation energy, if the kinetic mechanism that drives the transformation is the same. Therefore, the breaking of 1 hydrogen bond on average per molecule, with an activation energy of $\Delta H_{a}=22 \mathrm{~kJ} \mathrm{~mol}^{-1}$, corresponds to a temperature of about $72-78 \mathrm{~K}$, in agreement with the boundary of the metastability zones of high and low-density amorphous ice (70-90 K).

The reordering from $I_{a} h$ to $I_{a} l$ starts at $38 \mathrm{~K}$, in a gradual warm-up, and proceeds gradually. The onset at $38 \mathrm{~K}$ suggests that the activation energy is lowered by some mechanism, and the gradual transition indicates that the effect is of a range of activation energies. This probably reflects the distribution of binding states in the ice. Indeed, MD models of vapordeposited low-density ice (Buch 1990; Zhang \& Buch 1990; Buch 1992) show many defects in this network, causing a range in bond coordination. The model low-density ice has a mean potential energy per molecule of $20.9 \mathrm{~kJ} \mathrm{~mol}^{-1}$, averaged over time and all molecules in a cluster, but a broad distribution extends from about 7 to $28 \mathrm{~kJ} \mathrm{~mol}^{-1}$ and peaks at 17.5 and $23.5 \mathrm{~kJ} \mathrm{~mol}^{-1}$. Alternatively, it has been shown that 5 coordination can lower the activation enthalpy by about $10 \mathrm{~kJ}$ mole $^{-1}$ (Sciortino et al. 1992). High-density amorphous ice is particularly rich in 5-coordinated water.

Exposure to the electron beam at temperatures below $70 \mathrm{~K}$ causes the ice to flow, although the $I_{a} h$ structure is preserved. The low viscosity of high-density ice suggests that it can easily restructure its lattice, like liquid water. In liquid water, 5coordination has been invoked to account for the easy restructuring, because it allows a lowering of the activation enthalpy (Sciortino et al. 1992). Five-coordination is less important in low-density ice, and absent in cubic ice, in agreement with the observed higher viscosity.

The mechanism of electron beam processing of $I_{c}$ into $I_{a} h$ is not yet well understood. Elastic collisions of electrons in the bulk are not important for displacements (Heide 1984) and, hence, for the formation of $\mathrm{I}_{\mathrm{a}} \mathrm{h}$. At a given dose, only about $2 \times 10^{-4}$ of all molecules are displaced. Ionization processes, in which a water molecule is dissociated $\left(\mathrm{H}_{2} \mathrm{O} \rightarrow \mathrm{H}+\mathrm{OH}\right)$, can be significant. For the given radiation dose, about $30 \%$ of all water molecules are dissociated. However, other processes 
may dominate (for example, the electronic or vibrational excitation of the inter- and intramolecular hydrogen bonds). Studies of the influence of photons on this process may reveal new information. Note, for example, that photons that excite a single $\mathrm{O}-\mathrm{H}-\mathrm{O}$ bending or a single $\mathrm{O}-\mathrm{H}$ stretching vibration (6.27 and $3.07 \mu \mathrm{m}$ ) carry as much energy as a typical hydrogen bond (19 and $39 \mathrm{~kJ} \mathrm{~mol}^{-1}$, respectively). Visible and nearinfrared photons carry more energy than this and may contribute to the structural transformation if absorbed.

\subsection{Time-Temperature Diagram}

It is reasonable to assume that activation enthalpy is a local phenomenon and activation enthalpies at other sites in the lattice are not much affected by a local restructuring. Given a range of activation enthalpies that determine the onset and the completion of the high- to low-density transition, a timetemperature diagram can be constructed (Fig. 8), according to which the induction time $(\Delta t)$ of each phase of the transition behaves according to (Schmitt et al. 1989)

$$
\Delta t(\mathrm{~s})=v_{0} \exp \left(\Delta H_{a} / R T\right) .
$$

The kinetics of all transitions in water are governed by the intermolecular vibration $\left(v_{0}=9 \times 10^{-15} \mathrm{~s}\right)$ or bending $\left(v_{0}=\right.$ $2.1 \times 10^{-14} \mathrm{~s}$ ) timescales of water. The uncertainty in the time axis of Figure 8 (the vertical shift) is about a factor of 4 , depending on the definition of the onset.

If $\Delta H_{a}$ is of order $22 \mathrm{~kJ} \mathrm{~mol}^{-1}$, it follows that high-density amorphous ice can persist on a timescale of $10^{5} \mathrm{yr}$ at temperatures below $22 \mathrm{~K}$, even in the absence of irradiation or charged particle bombardment. Similarly, the high-density ice can persist below temperatures of $19 \mathrm{~K}$ on the solar system timescale of $4.5 \times 10^{9} \mathrm{yr}$.

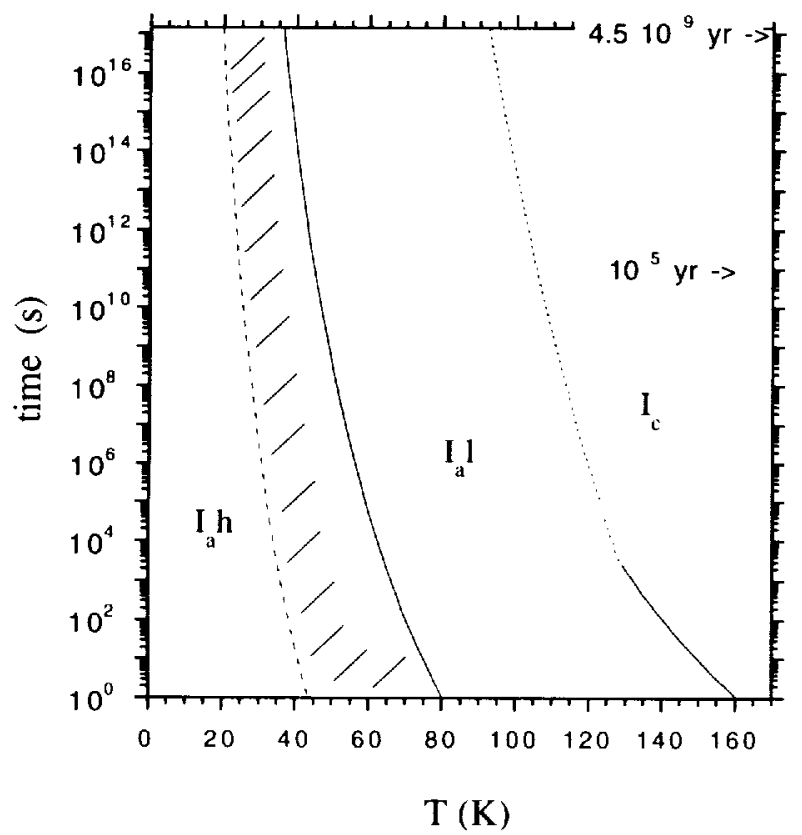

FIG. 8.-Time-temperature diagram, based on conjecture, showing the time it takes for the ice to start and complete the transformation from $\mathrm{I}_{\mathrm{a}} \mathrm{h}$ to $\mathrm{I}_{\mathrm{a}} \mathrm{I}$ after being warmed to a given temperature. The assumptions made are explained in the main text. Markers at $4.5 \times 10^{9}$ and $10^{5} \mathrm{yr}$ indicate the typical timescale of water-ice evolution in the solar system and in the dense interstellar medium, respectively

\section{ASTROPHYSICAL IMPLICATIONS}

\subsection{The $3.07 \mu \mathrm{m}$ Ice Band on Interstellar Dust Grains}

Ice in the interstellar medium is usually characterized by the strength and shape of the $3.07 \mu \mathrm{m}$ absorption band in the infrared (Knacke, Cudaback, \& Gaustad 1969; Gillett \& Forrest 1973; Merrill, Russell, \& Soifer 1976; Hagen et al. 1981; Whittet et al. 1983; Bult, Greenberg, \& Whittet 1985; Baratta \& Stazzulla 1990; Smith, Sellgren, \& Brooke 1993). This band is due to the $\mathrm{O}-\mathrm{H}$ stretch vibration of water. A unique assignment of shoulder and peaks of bands to vibrations is not possible because intra- and intermolecular coupling of $\mathrm{O}-\mathrm{H}$ oscillators are nearly equal in strength (Haas \& Hornig 1960; Bergren et al. 1978; McGraw et al. 1978). Incoherent inelastic neutron scattering gives a density of vibrational states that shows a strong difference between pressure-induced high- and low-density amorphous ices (hda and 1da) (Klug et al. 1991; Tse 1992). The strongest differences are in the longitudinal and transverse optic branches of the dispersion relation. The leading edge of the librational band begins at lower frequency in hda ice and rises more slowly. These differences are manifested in the band shapes of infrared absorption bands.

There is a clear difference in the $3.07 \mu \mathrm{m}$ band shape associated with vapor-deposited high- and low-density amorphous ice, $I_{a} h$, and $I_{a} l$. If the laboratory spectra of low-temperature deposits by Hagen et al. (1981) are indeed those of $I_{a} h$, as we believe they are, it follows that the $I_{a} h$ band contains additional absorption at high vibrational frequency. When the ice is warmed at a rate of less than $5 \mathrm{~K}$ minutes $^{-1}$, the peak wavelength changes from 3.04 to $3.08 \mu \mathrm{m}$, and the feature sharpens from 310 to $230 \mathrm{~cm}^{-1}$ in the temperature range $20-70 \mathrm{~K}$. The $45 \mu \mathrm{m}$ band is a less sensitive probe of the $I_{\mathrm{a}} \mathrm{h} \rightarrow I_{\mathrm{a}}$ I transition (Klug et al. 1991; Moore \& Hudson 1992).

Smith, Sellgren, \& Tokunaga (1989) found that some astrophysical objects show ice-band profiles that are best fitted by a combination of ice deposited at low temperature and warmed to 23,77 , and $150 \mathrm{~K}$. We now interpret these deposits as highdensity amorphous ice $I_{\mathrm{a}} \mathrm{h}$, low-density amorphous ice $I_{\mathrm{a}} \mathrm{l}$, and cubic crystalline ice $I_{c}$, respectively. For example, spectra from the Becklin-Neugebauer object are best fitted with spectral ratios of $I_{a} h: I_{a} 1: I_{c}$ equal to $3.1: 1.2: 1$, while Mon/IRS 3 is best fitted by ratios of 5.3:2.5:1 and GL 2591 by ratios of $8.6: 1.2: 1$. The $23 \mathrm{~K}$ ice band provides a satisfactory fit to the overall shape of most other known spectra. The conclusion must be that ice frozen on grains in the interstellar medium is principally in the high-density amorphous form.

The frost on interstellar grains constitutes the largest reservoir of ice in the universe - in our Galaxy alone about $10^{37} \mathrm{~kg}$ (Jenniskens et al. 1993). Little is known about extrasolar planetary systems, hence the amount of ice in icy planets and comets cannot be estimated with any certainty. However, if all stars have an amount of icy material in the form of icy planets, satellites and Kuiper Belt comets similar to that in our planetary system, then the total adds to perhaps $10^{30}-10^{31} \mathrm{~kg}$. We cannot think of other sources of ice. Hence, we conclude that the frost on interstellar grains is the largest reservoir of ice, and most ice in the universe is, therefore, in the high-density amorphous form.

\subsection{Formation of Icy Mantles}

The physical conditions present in molecular clouds are consistent with the presence of $I_{a} h$ instead of $I_{a} l$. The deposition 
rate of water vapor in dense clouds on $10 \mathrm{~K}$ cold silicate grains can be as low as $2 \times 10^{-11} \mu \mathrm{m} \mathrm{hr}^{-1}$, assuming that a typical ice layer of $0.02 \mu \mathrm{m}$ is grown in $10^{5} \mathrm{yr}$ (Jenniskens et al. 1993). Extrapolation of experimental data shows that, in spite of this very low deposition rate, no $I_{c}$ crystals form at substrate temperatures below $66 \mathrm{~K}$, given the activation energy involved in the $I_{a} l \rightarrow I_{c}$ transition. If, similarly, some activation energy applies for the $I_{a} h \rightarrow I_{a} l$ transition, it can be calculated whether $I_{a} h$ or $I_{a} l$ forms. For an activation enthalpy of $12 \mathrm{~kJ}$ mol $^{-1}, I_{a} h$, and not $I_{a} l$, is formed for any substrate temperature below $30 \mathrm{~K}$. Hence, evaporation and recondensation of icy mantles in dense cloud environments results in $\mathrm{I}_{\mathrm{a}} \mathrm{h}$ ice mantles, as observed.

Initially, however, the ice mantle is not formed by direct vapor deposition but by surface reactions of atomic oxygen and hydrogen: $\mathrm{O}+\mathrm{H} \rightarrow \mathrm{OH}$ and $\mathrm{OH}+\mathrm{H} \rightarrow \mathrm{H}_{2} \mathrm{O}$. In some environments, this initial ice layer may be preserved. One wonders if that ice would be structurally different from vapordeposited amorphous ice. The energy released in these surface reactions is on the order of $1 \mathrm{eV}$, which will locally heat the surface. Kouchi et al. (1994) argue that the resulting temperature spike decreases rapidly with increasing distance from the formation point, sufficiently fast to disallow the water molecules from setting into a crystalline structure. But does this prevent the formation of $I_{a} l$ instead of $I_{a} h$ ? It probably does. We point to the analogy with electron bombardment, where the input of energy in the ice matrix at $T<70 \mathrm{~K}$ results in high-density amorphous ice. Hence, if the release of the binding energy of $\mathrm{O}-\mathrm{H}$ chemical bonds has an effect similar to that of the deposition of energy by electrons or protons, then the ice is also expected to be distorted into the $I_{\mathrm{a}} \mathrm{h}$ form during initial formation of the water mantle at low temperature.

\subsection{CO as a Tracer of Structural Changes in the Water Matrix}

Impurities in the water ice matrix can be used as probes of the structure of the water mantle (Grim \& Greenberg 1987). The band positions of $\mathrm{CO}$ and $\mathrm{CO}_{2}$ have been studied in the laboratory (Sandford et al. 1988; Schmitt et al. 1989; Sandford \& Allamandola 1990; Palumbo \& Strazzulla 1993). Both bands are readily observable in astrophysical ices. Notably the $4.7 \mu \mathrm{m}$ band of $\mathrm{C}=\mathrm{O}$ has been studied using ground-based infrared telescopes (Whittet et al. 1983; Lacy et al. 1984; Kerr, Adamson, \& Whittet 1993; Chiar et al. 1994).

Two absorption maxima were identified in laboratory studies of $\mathrm{CO}: \mathrm{H}_{2} \mathrm{O}$ 1:20 mixtures at 10 K (Sandford \& Allamandola 1988). The main absorption is at $2137 \mathrm{~cm}^{-1}$, which is shifted to lower frequency (i.e., a repulsive bond) with respect to the position of pure $\mathrm{CO}$ at $2143 \mathrm{~cm}^{-1}$. In addition, there is a shoulder at $2152 \mathrm{~cm}^{-1}$ (an attractive bond) in fresh deposits at $T<40 \mathrm{~K}$, which accounts for $22 \%-26 \%$ of the total band intensity below $35 \mathrm{~K}$. The latter band progressively disappears (irreversibly) when the ice is warmed above $40 \mathrm{~K}$. The CO loss stops immediately when warm-up is stopped (Sandford \& Allamandola 1988), which implies that the loss of $\mathrm{CO}$ is controlled by changes in the water structure and that these structural changes are gradual. Indeed, the temperature zone where this band disappears (40-60 K at $2 \mathrm{~K}_{\text {minutes }}{ }^{-1}$ warm-up) coincides with our measured transition $\mathrm{I}_{\mathrm{a}} \mathrm{h} \rightarrow \mathrm{I}_{\mathrm{a}} \mathrm{l}$.

The peak at $2152 \mathrm{~cm}^{-1}$ was assigned to interstitial $\mathrm{CO}$ by Sandford \& Allamandola (1988), and the peak at $2137 \mathrm{~cm}^{-1}$ was thought to be due to $\mathrm{CO}$ that substitutes for a water molecule in the water-ice network. It is unlikely that $\mathrm{CO}$ can substitute for water in a water matrix, particularly at tem- peratures above $60 \mathrm{~K}$, and that upon warming from low temperatures, interstitial $\mathrm{CO}$ evolves into a substitutional site. Schmitt et al. (1989) and Grim (1988) assigned the two peaks to two types of water-CO interactions within the incompletely hydrogen-bonded network. While the $2137 \mathrm{~cm}^{-1}$ peak was thought to be due to unbonded $\mathrm{CO}$, the $2152 \mathrm{~cm}^{-1}$ peak was assigned to $\mathrm{CO}-\mathrm{H}_{2} \mathrm{O}$ hydrogen-bonded complexes in a water ice. This complex has a peak at $2149 \mathrm{~cm}^{-1}$ in an argon matrix (Dubost 1976) and $2146.5 \mathrm{~cm}^{-1}$ in a CO ice (Hagen \& Tielens 1981). During warm-up from 10 to $40 \mathrm{~K}$, the hydrogen-bonded complexes were thought to be preferentially replaced by hydrogen bonds of the normal water network. We believe this interpretation to be essentially correct. In light of our observation that the $2152 \mathrm{~cm}^{-1}$ peak is linked to $\mathrm{I}_{\mathrm{a}} \mathrm{h}$ ice, we propose that this peak is due to $\mathrm{CO}-\mathrm{H}_{2} \mathrm{O}$ complexes involving dangling $\mathrm{OH}$ bonds, while the $2137 \mathrm{~cm}^{-1}$ peak is due to $\mathrm{CO}$ trapped in "interstitial" sites in the ice cages, noting that such cages in $I_{\mathrm{a}} \mathrm{h}$ have a broader range of shapes and are typically smaller than those in $I_{a}$ l. This can account for the small shift to lower frequency and the slight narrowing of the $\mathrm{C}=\mathrm{O}$ stretch vibration during the $I_{a} h \rightarrow I_{a} l$ transition (Sandford \& Allamandola 1988).

The $\mathrm{CO}$ peak at $2152 \mathrm{~cm}^{-1}$ is absent in interstellar ices. This implies that the CO-containing ice mantles were warmed above $60 \mathrm{~K}$ on timescales less than that for water mantle evaporation. Indeed, most of the $\mathrm{CO}$ is found in more volatile apolar mantles. However, the water ice itself is not found in the $I_{a} l$ form. This observation suggests that $I_{a} l$ is transformed back into $I_{a} h$ on short timescales. The most likely transformation mechanism is photolysis. The photon flux in the dense cloud medium is high enough to allow a restructuring into the $\mathrm{I}_{\mathrm{a}} \mathrm{h}$ form on a timescale of less than $10^{5} \mathrm{yr}$, when $1 \%-10 \%$ of water molecules absorb a $10 \mathrm{eV}$ photon. Many more lower energy photons are absorbed. This cycling of $I_{a} h \rightarrow I_{a} l$ ice and back can play an important role in the generation of organic compounds of large molecular weight through UV photolysis, as long as traces of $\mathrm{CO}$ or $\mathrm{CO}_{2}$ remain in the ice (Jenniskens et al. 1993; Jenniskens \& Blake 1994). The photolysis will also cause the ice to be less viscous and form a smooth mantle around the ice grains. Hence, the effective surface area for $\mathrm{H}_{2}$ formation is probably not much different from that of a homogeneous surface.

\section{CONCLUSIONS}

The observed changes of the diffraction pattern of vapordeposited water ice as a function of temperature, the presence of two stability regimes for electron bombardment, and the similar rdf compared to the pressure-induced high- and lowdensity amorphous forms, among other arguments, leave no doubt as to the existence of high-density amorphous ice in low-temperature vapor deposits. It is a naturally occurring form which arises when water ice is slowly $\left(<100 \mu \mathrm{m} \mathrm{hr}^{-1}\right)$ vapor-deposited on any substrate of good thermal conductivity at temperatures $T<30 \mathrm{~K}$.

The radial distribution function of high-density amorphous ice, obtained by Fourier transformation of the diffraction patterns, has oxygen-oxygen distances between 3 and $4 \AA$, between the first and second nearest neighbor distance in low-density amorphous ice. The shape of the rdf is similar to that of pressure-induced hda reported by Bizid et al. (1987).

MD calculations can reproduce this $3-4 \AA$ feature, if the water ensemble is allowed to relax in a confined space. The 
feature is not due to unbonded interstitial water molecules. Four-coordination is still preferred, although in comparison to the low-density amorphous ice model, there is a somewhat larger number of five-coordinated water molecules. The most striking feature of the high-density amorphous structure is the small probability of finding cavities as large as those found in the two crystalline forms of ice, while low-density amorphous ice has a cavity size distribution more characteristic of an open crystalline structure (Fig. 7). From these results, we conclude that the high-density amorphous form is best described as a (partially) collapsed low-density ice structure.

There is an irreversible first-order transition from high- to low-density amorphous ice. Below $70-80 \mathrm{~K}$, there is a range of activation energy barriers that hinder restructuring. Several processes can transform the low-density amorphous form (or the crystalline cubic polymorph) back into high-density amorphous ice in this regime. These include electron and proton bombardment, pressure melting, and possibly UV photolysis. From similarities between the resulting radial distribution functions, and from results of MD simulations of pressure-induced amorphous ice in the literature, we infer that all of these processes cause some form of collapse of the lowdensity amorphous ice structure. During vapor deposition, the collapse of the water lattice may be a consequence of a rapid dissipation of the energy of binding before impinging molecules can reorient to form low-density amorphous ice. Alternatively, the ice may form a low-density amorphous ice structure initially, but then be distorted by the release of binding energy (analogous to distortions induced by electron irradiation).

The nature of the energy barrier determines the physical difference between the two amorphous forms. We propose that the restructuring of high-density ice into low-density ice requires the breaking of one hydrogen bond, on average, per molecule. The wide range of activation energies present at different locations in the vapor-deposited high-density amorphous ice may be responsible for the early onset. Alternatively, high-density ice does have a relatively large fraction of fivecoordinated water molecules, and there has been a suggestion that five-coordination is responsible for the lowering of the activation enthalpy needed for the rapid restructuring in liquid water.

Given the astrophysical conditions in the dense interstellar medium, we argue that vapor deposition on interstellar grains results in a water frost with high-density amorphous structure. We surmise that water mantles formed through the recombination of $\mathrm{H}$ and $\mathrm{OH}$ radicals result also in a high-density ice structure. A brief warming above $40 \mathrm{~K}$, however, will lead to a low-density amorphous form in the absence of sufficient irradiation processes. The structural transition from one amorphous form into another can play an important role in dynamic processes like radical diffusion and outgassing.

Ground-based observations of the $\mathrm{O}-\mathrm{H}$ stretch vibration at $3.07 \mu \mathrm{m}$ in a variety of astrophysical environments have been interpreted in terms of a composite of ice deposited at low temperature and warmed to 23,77 , and $150 \mathrm{~K}$. We now interpret these deposits as high-density amorphous ice $\left(I_{a} h\right)$, lowdensity amorphous ice $\left(I_{a} l\right)$, and cubic crystalline ice $\left(I_{c}\right)$, respectively. Most of the observed $3.07 \mu \mathrm{m}$ absorption bands in dense molecular clouds are best represented by a low- $T(<30$ K) deposit (Hagen et al. 1981) and, therefore, by the highdensity form of amorphous water ice. The frost on interstellar grains constitutes the largest reservoir of ice in the unverse-in our Galaxy alone, an estimated $10^{37} \mathrm{~kg}$ - and we therefore conclude that most ice in the universe is in this high-density amorphous form.

In the near future, the Infrared Space Observatory will offer a unique means to measure the structural state of water ice in different astrophysical environments, either directly, from the shape of the infrared absorption and emission bands of the vibration and bending modes of water, or indirectly, from the behavior of trapped impurities in the water matrix.

We thank G. Palmer, who is responsible for a number of important modifications to the electron microscope that made this work possible, and A. Breon, who automated the reduction process of large batches of diffraction patterns. The paper benefited from discussions with A. G. G. M. Tielens. Computer facilities were provided in part by the Numerical Aerodynamic Simulator (NAS) at NASA/Ames Research Center. This work was supported by a Director's Discretionary Fund proposal from NASA/Ames Research Center and grants from the Exobiology and Planetary Materials and Geochemistry Programs of the National Aeronautics and Space Administration. The research was performed while $P$. J. held a National Research Council-Ames Research Center Research Associationship.

\section{REFERENCES}

Angell, C. A 1983 Ann. Rev. Phys Chem 34,593

Bar-Nun, A., Dror, J., Kochavi, E., \& Laufer, D. 1987, Phys. Rev. B, 35, 2427

Bar-Nun, A., Herman, G., \& Laufer, D. 1985, Icarus, 63, 317

Baratta, G. A., \& Stazzulla, G. 1990, A\&A, 240, 429

Bergren, M. S., Schuh, D., Sceats, M. G., \& Rice, S. A. 1978, J. Chem. Phys., 69, 3477

Bizid, A., Bosio, L., Defrain, A., \& Oumezzine, M. 1987, J. Chem. Phys., 87, 2225

Blake, D. F., \& Palmer, G. 1991, in 26th Microbeam Analysis Society Meeting, ed. Hewitt D. G. (San Francisco: San Francisco Press), 293

Bosio, L., Johari, G. P., \& Teixeira, J. 1986, Phys. Rev. Lett., 56, 460

Brown, W. L., Augustyniak, W. M., Lanzerotti, L. J., Johnson, R. E., \& Evatt, R. 1980, Phys. Rev. Lett., 45, 1632

Brown, W. L., Lanzerotti, L. J., Poate, J. M., \& Augustyniak, W. M. 1978, Phys. Rev. Lett. 40, 1027

Buch, V. 1990, J. Chem. Phys., 93, 2631

Buch, 1992, J. Chem. Phys., 96, 3814

Burton, E. F., \& Oliver, W. F. 1935, Proc. R. Soc. Lond. A, 153, 166

Chiar, J. E., Adamson, A. J., Kerr, T. H., \& Whittet, D. C. B. 1994, ApJ, 426, 240

Dore, J., \& Teixeira, J. 1991, Hydrogen Bonded Liquids (Dordrecht: Kluwer)

Dubost, H. 1976, Chem. Phys., 12, 139
Fujime, S. 1966, Japanese J. Appl. Phys., 5, 764

Ghormley, J. A. 1967, J. Chem. Phys., 46, 1321 . 1968, J. Chem. Phys., 48, 503

Gillett, F. C., \& Forrest, W. J. 1973, ApJ, 179, 483

Grim, R. J. A., \& Greenberg, J. M. 1987, A\&A, 181, 155

. 1988, Ph.D. thesis, Leiden Univ.

Haas, C., \& Hornig, D. F. 1960, J. Chem. Phys., 32, 1763

Hagen, W., \& Tielens, A. G. G. M. 1981, J. Chem. Phys., 75, 4198

Hagen, W., Tielens, A. G. G. M., \& Greenberg, J. M. 1981, Chem. Phys., 56, 367

1983, A\& AS, 51, 389

Handa, Y. P., Mishima, O., \& Whalley, E. 1986, J. Chem. Phys., 84, 2766

Heide, H. G. 1984, Ultramicroscopy, 14, 271

Heide, H. G., \& Zeitler, E. 1985, Ultramicroscopy, 16, 151

Hemley, R. J., Chen, L. C., \& Mao. H. K. 1989, Nature, 338, 638

Jenniskens, P., Baratta, G. A., Kouchi, A., de Groot, M. S., Greenberg, J. M., \&

Strazzulla, G. 1993, A\&A, 273, 583

Jenniskens, P., \& Blake, D. F. 1994, Science, 265, 753

Kerr, T. H., Adamson, A. J., \& Whittet, D. C. B. 1993, MNRAS, 262, 1047

Klug, D. D., Whalley, E., Svensson, E. C., Root, J. H., \& Sears, V. F. 1991 Phys. Rev. B, 44, 841

Knacke, R. F., Cudaback, D. D., \& Gaustad, J. E. 1969, ApJ, 158, L51 
Kouchi A 1990 J. Cryst Growth 99, 1220

Kouchi, A., \& Kuroda, T. 1990, Nature, 344, 134

Kouchi, A., Yamamoto, T., Kozasa, T., Kuroda, T., \& Greenberg, J. M. 1994, A\&A, 290,1009

Lacy, J. H., Baas, F., Allamandola, L. J., Persson, S. E., McGregor, P. J., , Geballe, T. R. \& van der Bult, C. E. P. 1984, ApJ, 276, 533

Laufer, D., Kochavi, E., \& Bar-Nun, A 1987, Phys. Rev., B, 36, 9219

Lepault, J., Booy, F. P., \& Dubochet, J. 1983, J. Microsc., 129, 89

Mayer, E., \& Pletzer, R. 1985, J. Chem. Phys., 83, 6536 1986, Nature, 319, 298

McGraw, R., Madden, W. G., Bergren, M. S., Rice, S. A., \& Sceats, M. G. 1978. J. Chem. Phys., 69, 3483

Merrill, K. M., Russell, R. W., \& Soifer, B. T. 1976, ApJ, 207, 763

Mishima, O. Calvert, L. D., \&Whalley, E. 1984, Nature, 310,393

- 1985, Nature, 314,76

Moine, P., Pelton, A. R., \& Sinclair, R. 1988, Journal of Non-crystalline Solids 101,213

Moore, M. H., Ferrante, R. F., Hudson, R. L., Nuth, J. A., \& Donn, B. 1994 ApJ, 428, L8

Moore M.H \& Hudson, R. L. 1992, ApJ, 401, 353

Mumma, M. J Weissman, P. R. \& Stern, S. A. 1993, in Protostars and Planets

III, ed. E. H. Levy, J. I. Lunine, \& M. S. Matthews (Tucson: Univ. Arizona Press), 1177

Narten, A H Venkatesh, C. G. \& Rice, S. A. 1976, J. Chem. Phys., 64, 1106

Okabe, H. 1978, Photochemistry of Small Molecules (New York: Wiley

Interscience)

Olander, D., \& Rice, S. A., 1972, Proc. Natl. Acad. Sci., 69, 98

Palumbo, M. E., \& Strazzulla, G. 1993, A\&A, 269, 568

Pohorille A Pratt, L. R., LaViolette, R. A., Wilson, M. A.. \& MacElroy, R. D. 1987, J. Chem. Phys., 87, 6070

Poole, P. H., Sciortino, F., Essmann, U., \& Stanley, H. E. 1992, Nature, 360, 324

Pratt, L. R. \& Pohorille, A. 1992, Proc. Natl. Acad. Sci., 89, 2995

Pitzhaupt G Smyrl N \& Devlin, J. P 1976, J. Chem. Phys., 64, 435

Sandford, S \& Allamandola, L. J, 1988, Icarus, 76, 201

Sandford, S. A., \& Allamando

Sandford, S. A., Allamandola, L. J., Tielens, A. G. G. M., \& Valero, G. J. 1988, ApJ, 329, 498
Sceats, M. G., \& Rice, S. A. 1982, in Water: A Comprehensive Treatise, ed. F. Franks (New York: Plenum), 83

Schmitt, B. Espinasse, S. Grim. R. J. A., Greenberg, J. M., \& Klinger, J. 1989 in Laboratory Studies of Cometary Ice Analogues, ed. J. Hunt \& T. D. Guyenne (Paris: ESA), 65

Schmitt, B. Greenberg, J.-M., \& Grim, R. J. A. 1989, ApJ, 340, L 33

Schmitt, B. Ocampo, J., \& Klinger, J. 1987, J. de Phys. III, 48, 519

Schutte W A 1988, The Evolution of Interstellar Organic Grain Mantles. Ph.D. thesis, Leiden Univ., The Netherlands

Schutte, W. A., Allamandola, L. J., \& Sanford, S. A. 1993, Science, 259, 1143

Sciortino, F., Geiger, A., \& Stanley, H. E. 1992, J. Chem. Phys., 96, 3857

Sivakumar, T. C., Rice, S. A., \& Sceats, M. C. 1978, J. Chem. Phys., 69, 3468

Sivakumar, T. C. Schuh. D., Sceats, M. G., \& Rice, S. A. 1977, Chem. Phys. Lett., 48, 212

Smith, R. G., Sellgren, K., \& Brooke, T. Y. 1993, MNRAS, 263, 749

Smith, R. G., Sellgren, K., \& Tokunaga, A. T. 1989, ApJ, 344, 413

Sugisaki, M. Suga, H., \& Seki, S. 1968, Bull. Chem. Phys. Soc. Japan, 41, 2501

Tielens, G G M \& Allamandola, L J 1987, in Physical Processes in Interstellar Clouds, ed. G. E. Morfill \& M. Scholer (Dordrecht : Reidel), 333

Tielens, A. G. G. M., Hagen, W., \& Greenberg, J. M. 1983, J. Phys. Chem., 87, 4220

Tse, J. 1992, J. Chem. Phys., 96, 5482

Tse J. S \& Klein, M. L. 1987, Phys. Rev. Lett., 58, 1672 1990, J. Chem. Phys., 92, 3992

Tse, J. S., Tan, K. H., \& Chen, J. M. 1990, Chem. Phys. Lett., 174, 603 van der Bult, C. E. P. M., Greenberg, J. M., \& Whittet, D. C. B. 1985, MNRAS, 214,289

Venkatesh, C. G., Rice, S. A.. \& Bates, J. B. 1975, J. Chem. Phys., 63, 1065

Westley, M. S., Baragiola, R. A., \& Johnson, R. E. 1995, Nature, 373,405

Whittet, D. C. B., Bode, M. F., Longmore, A. J., Baines, D. W. T., \& Evans, A 1983, Nature, 303, 218

Wilson, M. A Pohorille, A., Jenniskens, P., \& Blake, D. F. 1995, Origins of Life, 25,3

Zallen, R. 1983, The Physics of Amorphous Solids (New York: Wiley)

Zhang, Q., \& Buch, V. 1990, J. Chem. Phys., 92, 5004 
